\title{
ESBC: an Enhanced Modular Multilevel Converter with H-Bridge Front End
}

\author{
Emmanuel Amankwah \\ The University of \\ Nottingham \\ Nottingham, UK \\ emmanuel.amankwah@ \\ nottingham.ac.uk
}

\author{
Alessandro Costabeber \\ The University of \\ Nottingham \\ Nottingham, UK \\ alessandro.costabeber@ \\ nottingham.ac.uk
}

\author{
Omar Jasim \\ GE Energy Connections \\ GE \\ Stafford, UK \\ Omar.Jasim@GE.com
}

\author{
David Trainer \\ GE Energy Connections \\ $\mathrm{GE}$ \\ Stafford, UK \\ David.Trainer@GE.com
}

\author{
Jon Clare \\ The University of \\ Nottingham \\ Nottingham, UK \\ jon.clare@nottingham.ac.
}

\begin{abstract}
This paper presents the Enhanced Series Bridge Converter (ESBC), a hybrid modular multilevel converter with $\mathbf{H}$ bridge front end suitable for high power grid applications. It retains the advantages of other modular multilevel topologies while offering compact structure, making it attractive for offshore stations, back-back HVDC stations, and city centre infeeds. The structure, operating principles and energy management of the converter are discussed. Simulation results from a scaled down medium voltage demonstrator are presented to validate the concept.
\end{abstract}

Keywords-Modular Multilevel Converters, HVDC Transmission, Offshore, H-bridge.

\section{INTRODUCTION}

Modular Multilevel Voltage Source Converters (MM-VSCs) have the inherent advantage of generating high quality voltage and current waveforms. These converters are also scalable making them more attractive for high voltage/power grid applications such as HVDC and FACTS. The efficiencies of HVDC installations using the MM-VSCs are comparable to that of more established HVDC technologies based on Line Commutated Converters (LCC) [1-4]. In recent times, many MM-VSC HVDC installations have been implemented [5-7]. However, there is significant research interest among academics and industry to develop an optimum HVDC VSC with high efficiency, high robustness to system faults and compact size while maintaining the other advantages of the modular VSC in power systems.

In $[1,8,9]$ different variants of the MM-VSCs exploiting an $\mathrm{H}$-bridge front end are investigated. This paper presents the Enhanced Series Bridge Converter (ESBC), which is the further development of the recently presented Series Bridge Converter (SBC) [8]. The topology is composed by the series connection on the DC side of three single phase units, each of them including a multilevel half-bridge arm (Chain-Link), two sets of multilevel full-bridge arms being deployed as series full bridge (SFB) arm and T-branch, H-bridge front end, and interface transformer. Ideally, on the DC side the three phases generate a set of phase shifted rectified sinewaves, adding to the DC voltage plus a $6 \mathrm{n}$ harmonics voltage ripple. The T-branch provides the harmonic filtering required to generate smooth DC voltage at the DC terminals. In the topology in [8], the voltage filtering is embedded in CLs and SFBs, thus influencing the inter-arm energy dynamics and therefore the amount of second harmonic required for the inter-arm energy management [8]. In the ESBC, the T-branch is independent from CLs and SFBs and it does not influence the energy management. Therefore, the second harmonic voltage needed for energy management can be reduced. This reduction helps to reduce the size of the SFB arms. In this paper, the general operation of the ESBC is presented. Control algorithm and energy management concepts are discussed and simulation results from a scaled down medium voltage demonstrator are presented.

\section{CONVERTER TOPOlOGY}

Figure 1 shows the two basic configurations of the ESBC. In these configurations, the T-branch is introduced to extract the 6n harmonic voltages across the DC bus. In Figure 1a, the Tbranch submodules are lumped together at the DC rail of the converter while in Figure $1 \mathrm{~b}$, the submodules are represented on a per phase basis. As can be observed from Figure 1a and Figure $1 b$, there is no significant difference in the performance of the T-branch during symmetrical network operating conditions, therefore in this paper all studies will be undertaken considering Figure 1a. Nevertheless, the studies and observations can easily be applied to Figure $1 \mathrm{~b}$ with the appropriate scaling. The converter now consists of two wave shaping arms, an H-bridge front end and a T-branch arm for harmonic voltage filtering. The shunt connected arm with halfbridge submodules is named Chain-Link (CL), while the series connected arm with full-bridge submodules is called Series Full Bridges arm (SFB). The newly introduced T-branch is a full bridge arm, which is in the main DC path. The CL and SFB arms are responsible for synthesising a variable amplitude full wave rectified sinusoidal voltage on the DC input of each $\mathrm{H}$ bridge, that 'unfolds' the waveform at the zero crossings of the voltage to generate the $\mathrm{AC}$ voltages across the primary side of three single phase open winding transformers as shown in Figure 1. An open winding transformer configuration is adopted to decouple the converter phase connection on the $\mathrm{AC}$ side. The three series connected CL units support the DC bus 


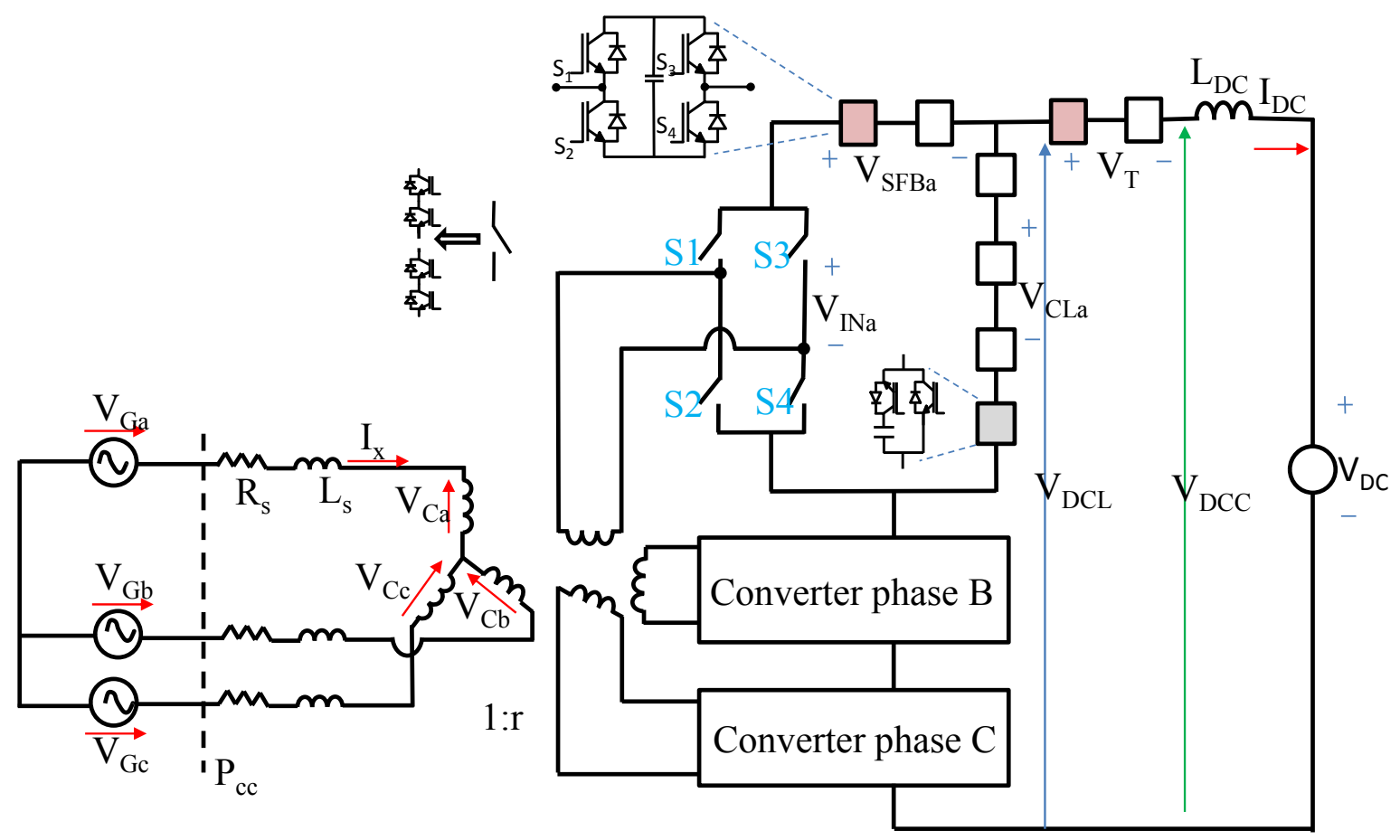

Figure 1a: Lumped T-branch

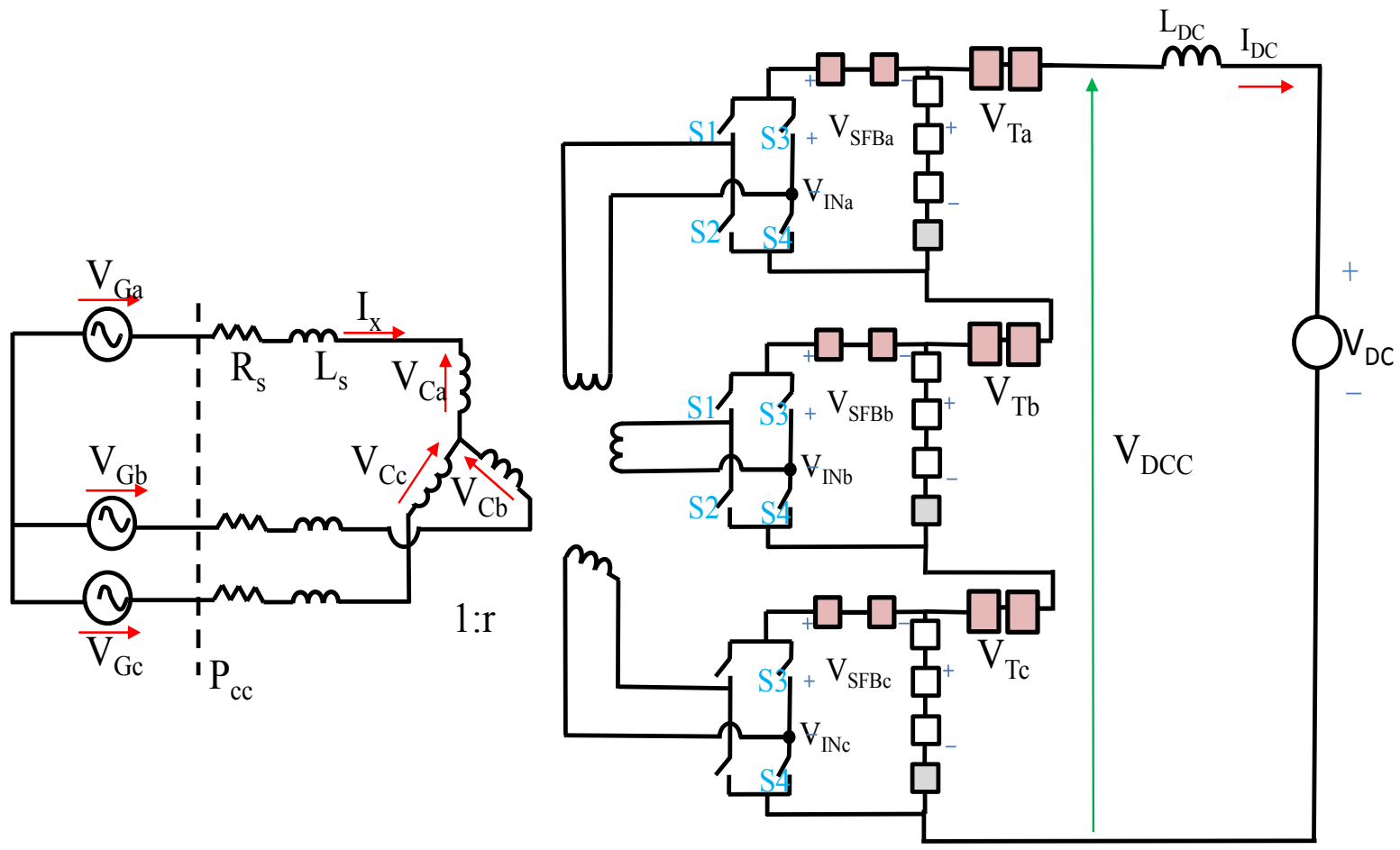

Figure 1b: Distributed T- Branch 
voltage while the three SFB units operate to decouple the AC network from the DC network and achieve full control of the AC side current and voltage. The configuration of the topology inherently improves the performance and size of the converter as:

- The converter consists of an optimal number of full and half bridge submodules which are less than that in the standard MMC either with half OR full bridge submodules.

- The DC current component in the half-bridge CLs is less than $20 \%$ of the rated DC current [1] and therefore the $\mathrm{CL}$ arms are less lossy.

- The power pulsation in the SFB arms has a fundamental frequency which is twice the line frequency and therefore the size of submodule capacitors can be reduced.

- The T-branch submodules capacitance is small as the lowest harmonic component synthesised by the branch is the $6^{\text {th }}$ order.

- The use of the T-branch means that the amount of second harmonic voltage required for inter-arm energy management as discussed in [8] is reduced and therefore the size of the SFB branch is also reduced.

Therefore, the converter can be made more compact and efficient compared to other similar MM-VSCs such as the standard MMC, the AAC and similar topologies. Although the $\mathrm{H}$-bridge based SFB arms and the H-bridge front ends are placed in the full current path, the number of submodules in the CL and the SFB arms can be optimally selected to achieve the required $\mathrm{PQ}$ operation while maintaining low conduction loss and compact size.

\section{OPERATING PRINCIPLE}

The operation of the ESBC can be discussed focusing on three main functions: (1) Support the DC bus voltage by the series connected CLs, (2) Formulation and "unfolding" of the full wave rectified voltages into AC voltages and currents, (3) Filtering of the resultant DC side harmonics caused by the operation of the CLs. As presented in Figure 1, the ESBC is composed of four elements in each phase: front-end H-bridge, SFB, CL and the T-branch. The H-bridge front end has the same task as in the SBC [8] of "unfolding" the full wave voltages imposed at the $\mathrm{DC}$ rail into $\mathrm{AC}$ waveforms at the converter $\mathrm{AC}$ terminals. The CLs synthesise full wave rectified sinusoidal voltages with a $\mathrm{DC}$ component matching the required $\mathrm{DC}$ bus voltage. The SFB branch then provides attenuation between the voltages synthesised by the $\mathrm{CL}$ and that required at the converter AC terminal to be able to achieve variable active power $(\mathrm{P})$ and reactive power $(\mathrm{Q})$ control. As shown in earlier publications of other embodiments of the SBC, the full wave rectified operation of the CL arms of the SBC results in $6 n$ voltage harmonics in the DC network. In the work presented in this paper, the T-branch is used to remove these harmonic components from the DC network.

In its basic form, the operation of the ESBC can be illustrated considering a balanced and stiff three phase AC network, with the impedances dominated by the leakage inductances of the transformers, and consider first an operation where the $\mathrm{AC}$ voltage can be matched with the CL. In this case, ideally, the SFB arms can be bypassed. In this condition, the three CLs generate three full wave rectified sinusoids displaced 120 electrical degrees. The turns ratio r: 1 of the transformer can be selected to match the amplitude of the converter voltage and grid voltage in this ideal condition. In this example, the voltage imposed by the grid, that to be synthesised by the converter, and that synthesised by the CL can be described for phase ' $a$ ' in (1), where $\delta$ is the phase shift between the grid voltage and the converter voltage and a corresponding phase shift $\varphi$ of the grid current.

$$
\begin{aligned}
& V_{G a}(t)=V_{G} \sin (\omega t), \quad V_{C a}(t)=V_{C} \sin (\omega t+\delta), \\
& I_{a}(t)=I \sin (\omega t+\varphi), \quad V_{I N a}=\left|V_{C a}(t)\right|, \\
& V_{S F B a}(t)=0, \quad V_{C L a}(t)=V_{I N a}
\end{aligned}
$$

Clearly, the average of the voltages in the three CLs can be shown to be (2):

$$
\bar{V}_{D C C}=\sum_{x=a, b, c} \bar{V}_{C L x}=3\left(V_{C} \frac{2}{\pi}\right)=V_{C} \frac{6}{\pi} \rightarrow V_{C}=\bar{V}_{D C C} \frac{\pi}{6}
$$

As stated earlier, in (1) and (2) the performance of the SFB arm and T-branch are not considered in the waveform formulation and therefore the amplitude of the converter $\mathrm{AC}$ voltages is directly related to the $\mathrm{DC}$ voltage and influences the selection of the turn ratio of the transformer. To decouple the peak converter voltage, $\mathrm{V}_{\mathrm{C}}$, from the composite $\mathrm{DC}$ voltage in the CLs, the SFB arms would be required to synthesise the residual voltage between the voltage required at the DC input of the H-bridge front-end, $\mathrm{V}_{\mathrm{INa}}(\mathrm{t})$ and that synthesised by the corresponding $\mathrm{CL}$ arm and allow for a system operation with a fixed transformer turns ratio. Consider now a more general case where the SFBs are inserted into the circuit. For a given $(\mathrm{P}, \mathrm{Q})$ operating point on the $\mathrm{AC}$ side, the amplitude $\mathrm{V}_{\mathrm{C}}$ and the phase shift $\delta$ of the converter voltages can be expressed as (3).

$$
\begin{aligned}
& V_{G a}(t)=V_{G} \sin (\omega t) \rightarrow V_{C}, \delta \rightarrow V_{C a}(t)=V_{C} \sin (\omega t+\delta), \\
& I_{a}(t)=I \sin (\omega t+\varphi)
\end{aligned}
$$

For a given active power $\mathrm{P}$, also the $\mathrm{DC}$ voltage demand of the converter $\mathrm{V}_{\text {DCC }}$ is defined. With $\mathrm{V}_{\text {DCC }}$, the amplitude of the rectified sinusoids forming the $\mathrm{CL}$ voltages can be derived. To limit the SFB voltage requirements, the rectified CL waveforms can be made proportional to the rectified AC voltage on the converter side. Referring to phase ' $a$ ': 
$V_{C L}=\frac{\pi}{6} V_{D C C} \rightarrow V_{C L a}(t)=V_{C L}|\sin (\omega t+\delta)|$

The difference required to generate $\mathrm{V}_{\mathrm{C}}$ is managed by the SFBs, guaranteeing reactive power control:

$V_{I N a}=V_{C}|\sin (\omega t+\delta)| \rightarrow V_{S F B a}(t)=\left(V_{C}-V_{C L}\right)|\sin (\omega t+\delta)|$

Fourier spectrum of the individual CL voltages contains even harmonics $2 \mathrm{n}$, that will cancel in the sum across the three phases except for those harmonics that are also multiples of 3 -i.e. the DC voltage will contain $6 \mathrm{n}$ ripple components (6). In this mode of operation, the SFBs are used to control reactive power but voltage ripple will appear in the DC circuit.

$V_{D L C}=\bar{V}_{D C C}+V_{R}(t)$

$V_{R}(t)=-\frac{12}{\pi} V_{C L} \sum_{n=1}^{\infty}\left(\frac{1}{(6 n)^{2}-1} \cos (6 n \omega t+6 n \delta)\right)$

In contrast to the operation of the SBC as presented in [8], the voltage harmonics $V_{R}(t)$ in (6) are extracted from the DC circuit using the newly introduced T-branch in Figure 1. In summary, the CL voltage, the SFB voltage, the input voltage for the main $\mathrm{H}$-bridge and the T-branch voltages that guarantees full power control and active DC filtering are:

$V_{C L a}(t)=V_{C L}|\sin (\omega t+\delta)|$

$V_{S F B a}(t)=\left(V_{C}-V_{C L}\right)|\sin (\omega t+\delta)|=\left(V_{C}-V_{C L}\right)|\sin (\omega t+\delta)|$

$V_{I N a}(t)=V_{C L a}(t)+V_{S F B a}(t)=V_{C}|\sin (\omega t+\delta)|$

$H$ bridge unfolding $\rightarrow V_{C a}(t)=V_{C} \sin (\omega t+\delta)$

$V_{T}(t)=-V_{R}(t)=\frac{12}{\pi} V_{C L} \sum_{n=1}^{\infty}\left(\frac{1}{(6 n)^{2}-1} \cos (6 n \omega t+6 n \delta)\right)$

The proposed methodology for voltage waveform formulation for CLs and SFBs minimises the impact of the SFBs on converter size and loss.

\section{ENERGY MANAGEMENT}

In this section, the impact of the considered waveform formulation on the energy distribution of the converter arms is investigated. Referring to Figure 1, consider the scenario where $\mathrm{x}=\mathrm{a}$ and the rectified sinusoid $\mathrm{V}_{\mathrm{IN}}$ is unfolded at the zero crossings we get:

$I_{S F B a}(t)=I_{a}(t) \operatorname{sign}\left(V_{C a}(t)\right)$

Considering again a generic operating point with $V_{C a}(t)=V_{C} \sin (\omega t+\delta), I_{a}(t)=I \sin (\omega t+\varphi) \quad$, the current through the corresponding CL arm can be described as (9)

$I_{C L a}(t)=I_{S F B a}(t)-I_{D C}$
By combining the voltages (7) and the corresponding currents (8) and (9), the average powers sourced/sinked by a CL and SFB can be expressed as (10):

$$
\begin{aligned}
\bar{P}_{C L a} & =-\frac{V_{D C C} I_{D C}}{3}+\frac{V_{C L} I}{2} \cos (\delta-\varphi) \\
& =-\frac{V_{D C C} I_{D C}}{3}+\frac{\pi V_{D C C} I}{12} \cos (\delta-\varphi)=-\bar{P}_{S F B a}
\end{aligned}
$$

Equation (10) can be conveniently rewritten including equation (4) and defining the DC power $P_{D C}=V_{D C C} I_{D C}$ :

$$
\begin{aligned}
\bar{P}_{C L a} & =-\frac{V_{D C C} I_{D C}}{3}+K_{U} \frac{V_{C} I}{2} \cos (\delta-\varphi) \\
& =-\frac{P_{D C}}{3}+\frac{V_{C L}}{V_{C}} P_{A C a}=-\bar{P}_{S F B a}
\end{aligned}
$$

Where $P_{A C a}$ is the active power absorbed by phase $a$. Average power into the T-branch of the converter can similarly be derived from (7) and considering the DC flow through the Tbranch as (12):

$$
\bar{P}_{T}=-V_{R}(t) I_{D C}=0
$$

Equation (11) shows that the average powers in the CL and SFB arms can be zero if $V_{C L}=V_{C}$ or if $\cos (\delta-\varphi)=0$ and $I_{D C}=0$, but generally $\bar{P}_{C L a}=-\bar{P}_{S F B a} \neq 0$. However, with the T-branch located in the DC circuit there is no net power exchange between the components of the arms other than the residual device losses that needs to be managed during converter operation. However, an active energy management is required to maintain the energy within the arms of the SFB and the CL. A method of using the $2^{\text {nd }}$ harmonic voltage injection to achieve this energy management has been considered in other publications on the SBC and will be adopted also in this work. However, the amount of $2^{\text {nd }}$ harmonic required is less compared to that used in the previous work as the filtering of the voltage harmonics using the T-branch does not affect the inter-arm energy as in [8]. This can be appreciated from the average power equation (10) where there is no effect of the $6 \mathrm{n}$ voltage harmonics in the average power in the $\mathrm{CL}$ and $\mathrm{SFB}$ arms. In the study of the SBC, however, the $6 n$ harmonic voltage affect significantly the energy variation in the arms of the SFB and the CL.

\section{Design AND Simulation}

In this section, plots illustrating the design of the converter and the simulation results demonstrating the performance of the converter are presented. The design of the CL, SFB and 


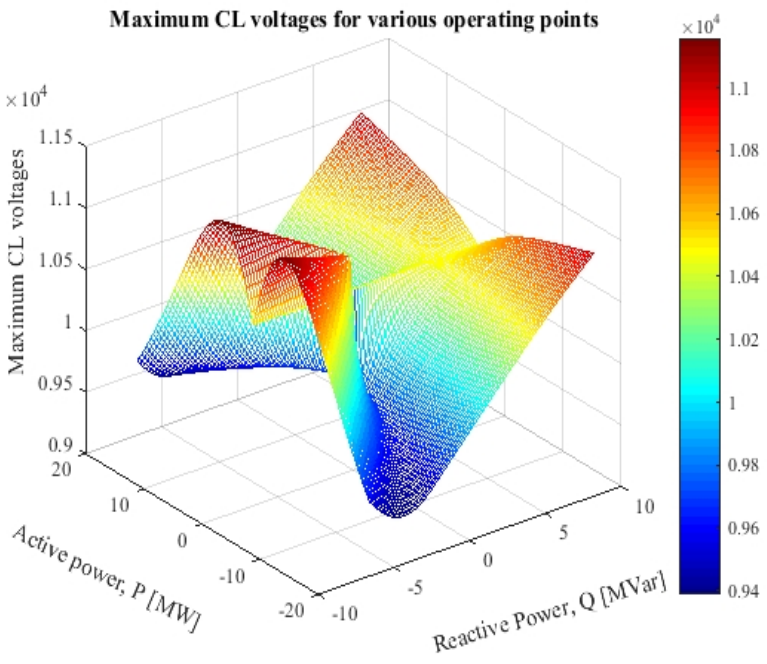

Fig. 2: Maximum CL Voltage required

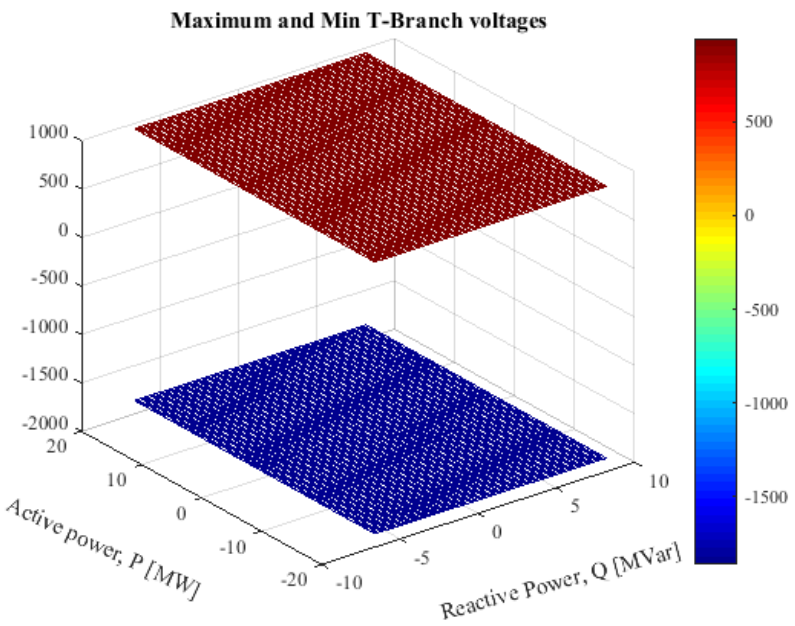

Fig. 4: Maximum and Minimum T-branch voltages

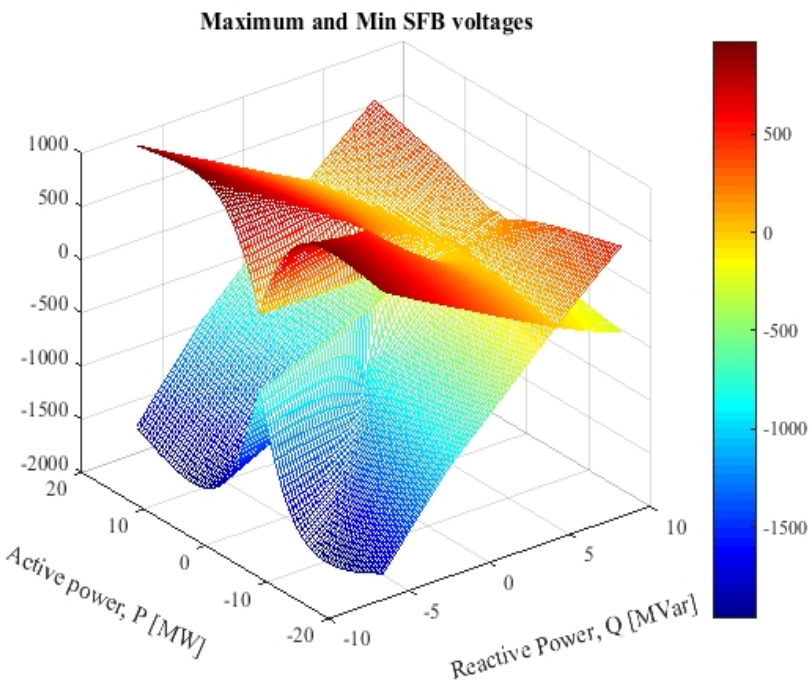

Fig. 3: Maximum and Minimum SFB voltages Injected second harmonic voltage for energy management

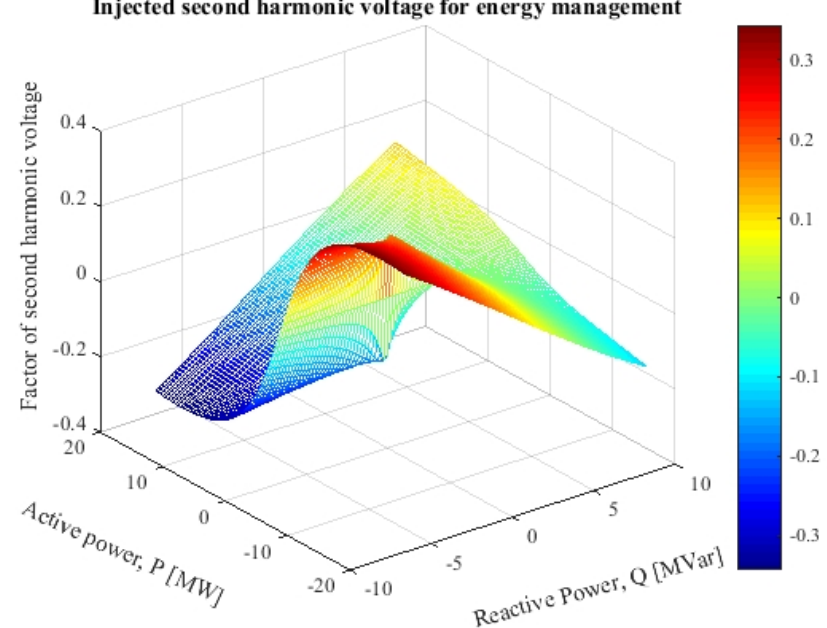

Fig. 5: Factor of second harmonic voltage required for inter arm energy management, i.e. amplitude of the second harmonic normalised by the peak of the $C L$ voltage before energy management in each operating point
T-branch arms are highly influenced by the voltages to be synthesised by these arms. Fig. 2 to Fig. 4 illustrate the voltage requirement for the various arms of the converter considering a $20 \mathrm{MW} / 20 \mathrm{kV} \mathrm{DC} / 11 \mathrm{kV}$ AC system. The factor of $2^{\text {nd }}$ harmonic voltage in the $\mathrm{CL}$ arm that will be required to manage power in the arms is presented in Fig. 5. The factor is defined as the ratio between the peak of the second harmonic voltage and the peak of the ideal CL voltage (i.e. without the need for second harmonic for energy management) in each operating point. Clearly, the rating of the T-branch voltages would have been extracted from the CL and be synthesised by the SFB, thereby increasing the size of the SFB and the amount of energy imbalance between the CL and SFB arms in the embodiments presented in [8]. Therefore, by using the T-branch, the amount of $2^{\text {nd }}$ harmonic voltage required for inter-arm energy management is reduced. In the T-branch, the predominant components are the $6^{\text {th }}$ harmonic components. This reduces drastically the size of the capacitors that are required to meet the peak to peak voltage ripple for the submodules in the Tbranch. From the plots in Fig. 2 to Fig. 4, a rating of $12 \mathrm{kV}$ for the $\mathrm{CL}$ arms, $3 \mathrm{kV}$ for the SFB arms and $3 \mathrm{kV}$ for the T-branch are considered for the $20 \mathrm{MW} / 20 \mathrm{kV}$ DC/ $11 \mathrm{kV} \mathrm{AC}$ demonstrator. A leakage inductance of 0.12PU typical of high power transformers is considered in this study. To maintain uniformity in the design of the submodules, local storage capacitors of $4 \mathrm{mF}$ operated at a nominal voltage of $1.5 \mathrm{kV}$ are used for all the arms. 

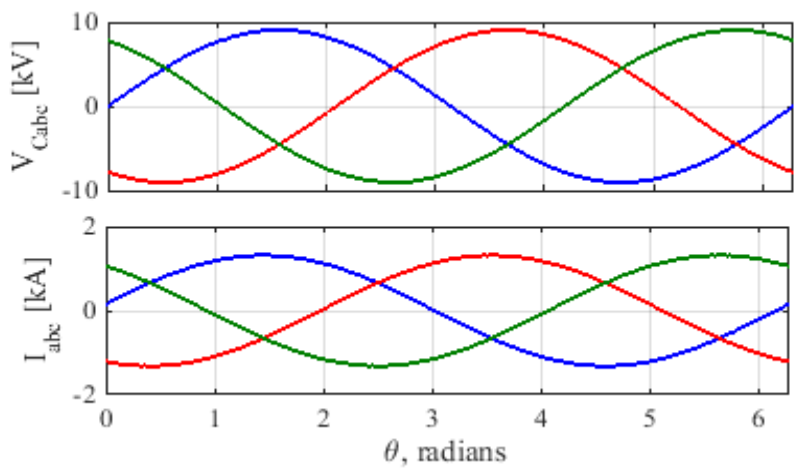

Fig. 6: Converter AC terminal voltages and currents, $\mathrm{V}_{\mathrm{G}}$ and $\mathrm{I}$
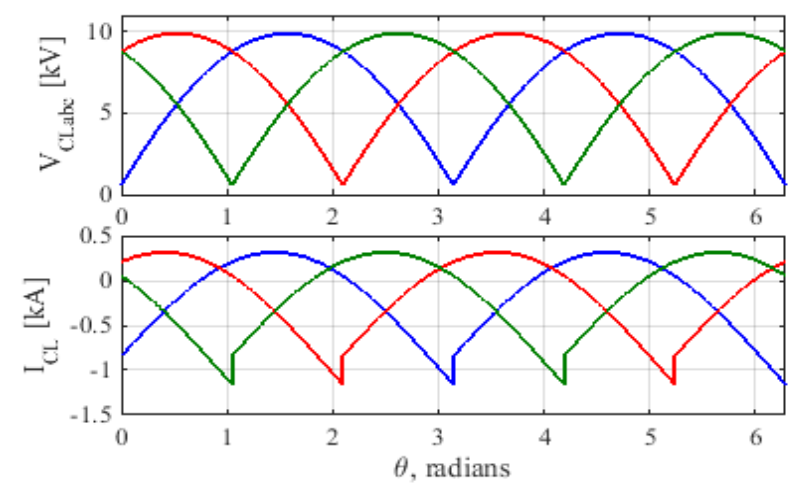

Fig. 8:CL voltages and currents
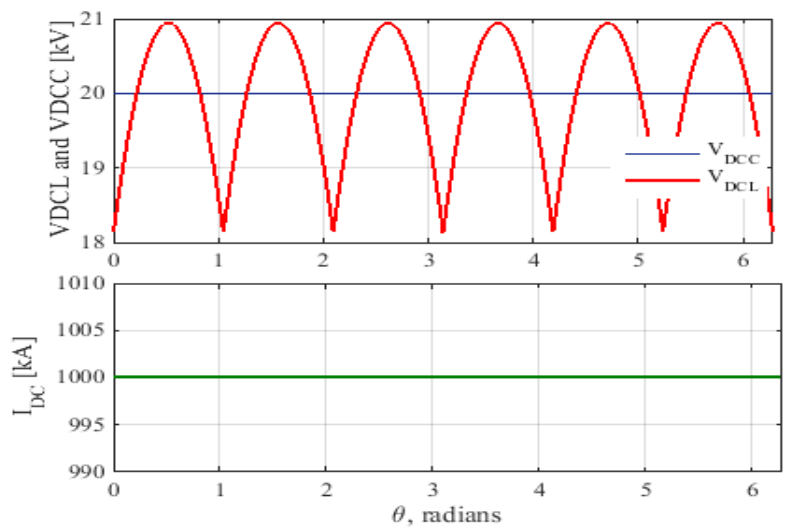

Fig. 10: Converter DC side voltages (with and without filtering) and DC network current

The operation of the converter can be illustrated considering Fig. 6 to Fig. 11. Consider that the voltages in Fig. 6 are imposed at the AC terminal of the ESBC, which will be rectified into full wave rectified sinusoidal voltages with the attendant current shaping as shown in Fig. 7. To help synthesise the required full wave voltage $\mathrm{V}_{\mathrm{IN}}$ and support the DC bus voltage, the $\mathrm{CL}$ arms synthesise the corresponding voltages as presented in Fig. 8. However, the voltages synthesised by the $\mathrm{CL}$ does not match that required at the DC input of the H-Bridge front-end, therefore the SFB arms synthesise the difference
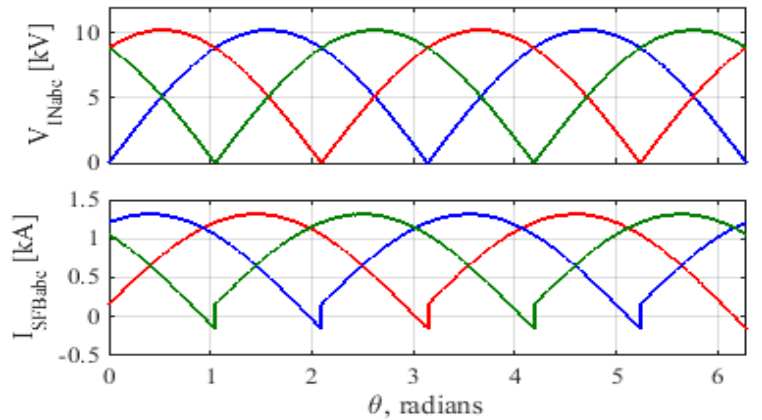

Fig. 7: Converter voltages and currents at DC input of the H-Bridge frontends

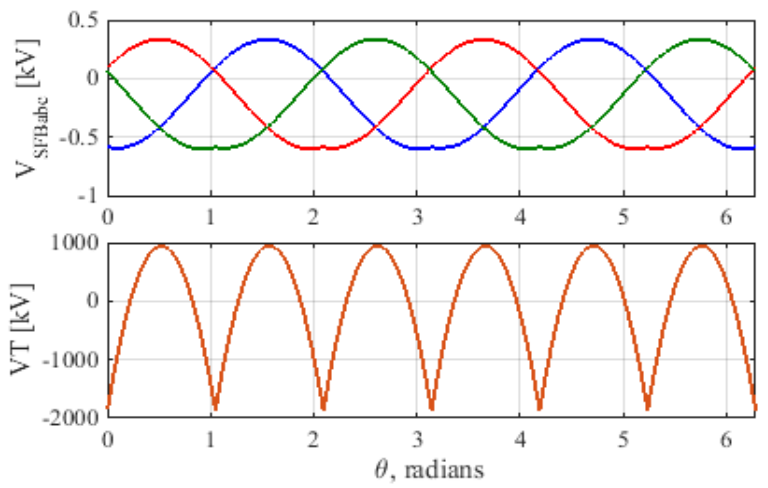

Fig. 9: SFB and T-branch voltages
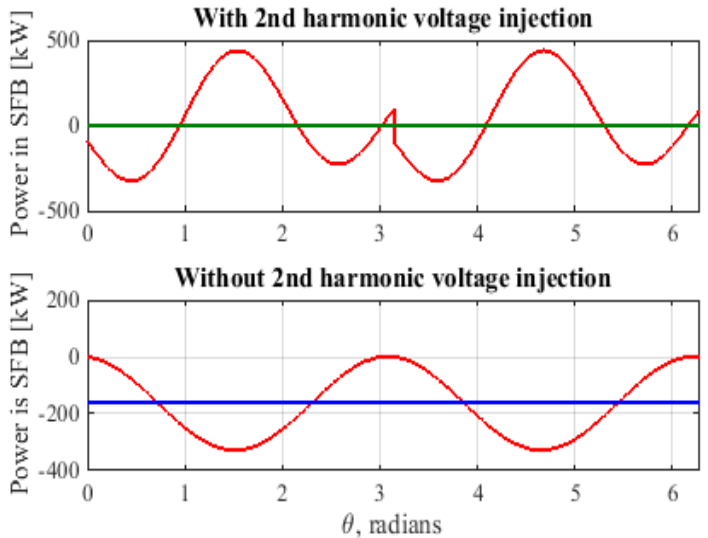

Fig. 11: Instantaneous and average power in the SFB arms with and without 2nd harmonic inter arm energy control

between that synthesised by the $\mathrm{CL}$ and the corresponding $\mathrm{V}_{\mathrm{IN}}$ as presented in Fig. 9. As shown earlier in (11) this results in residual powers in the SFB which will have to be managed by the use of the $2^{\text {nd }}$ harmonic voltage injection. Also, the operation of the $\mathrm{CL}$ also results in the $6 \mathrm{n}$ harmonic voltages in $\mathrm{V}_{\mathrm{DCL}}$ as shown in (6). The voltage synthesised by the SFB by supporting the $\mathrm{V}_{\mathrm{IN}}$ formation and inter-arm energy management are shown in Fig. 9. The voltage harmonics that are extracted from $V_{D C L}$ by the use of the T-branch is shown in lower plot of Fig. 9. Fig. 10 illustrates $V_{\text {DCL }}$ with the 6 n harmonic voltages 

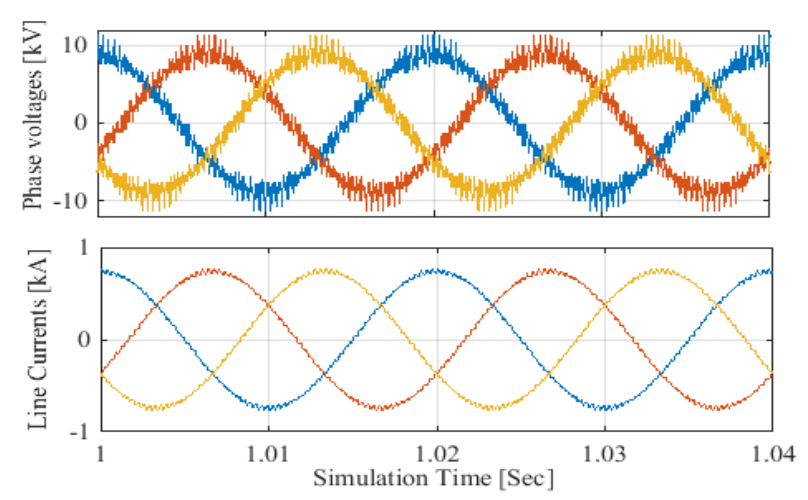

Fig. 12: Grid side voltages and Currents
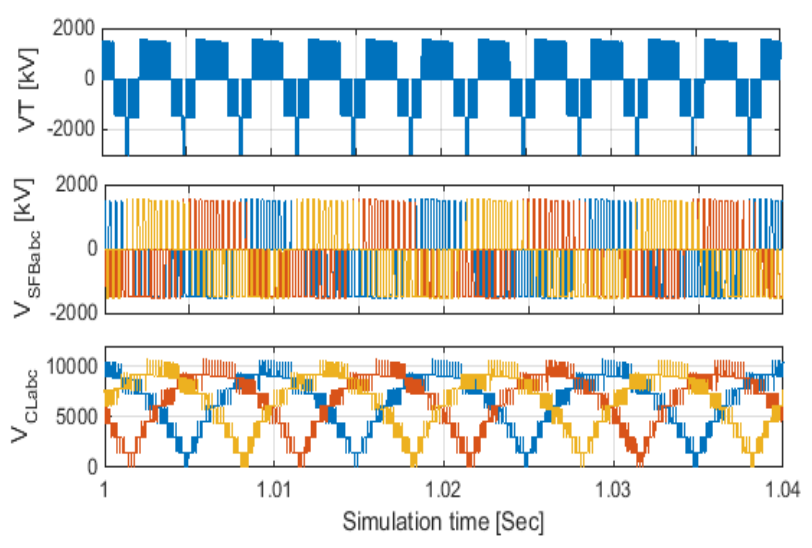

Fig. 14: Voltages formulated by the various arms of the ESBC

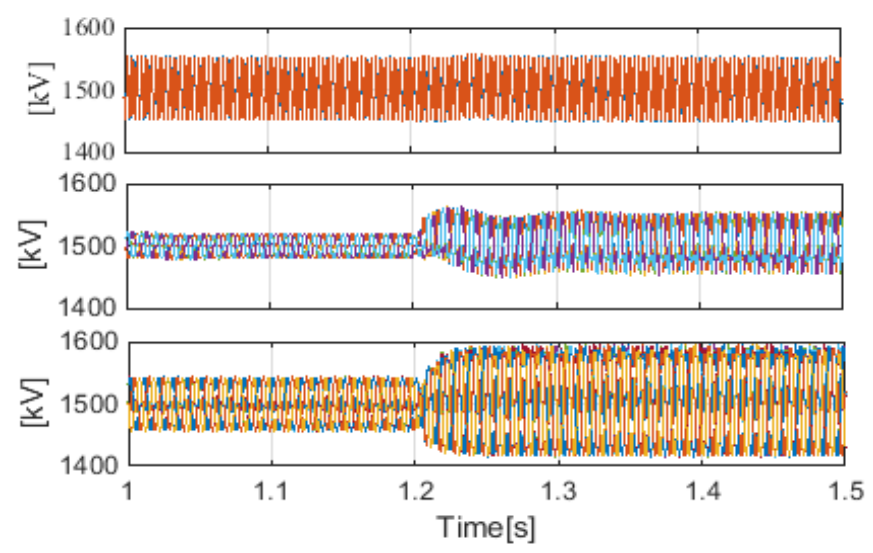

Fig. 16: Local submodule capacitor voltages in the various arms of the converter (top) T branch, (middle) SFB and (bottom) CL

and $\mathrm{V}_{\mathrm{DCC}}$ when the $6 \mathrm{n}$ harmonic voltages have been extracted using the T-branch, showing that the voltage seen by the DC network is ripple free. The effect of the energy management on the SFB performance is illustrated with Fig. 11. Clearly, it can be observed that the energy in the arms can be managed with the use of the second harmonic voltage injection.

Fig. 12 to Fig. 17 present waveforms from a detailed switching model of 20kVDC 20MW system with 8, 2 and 2
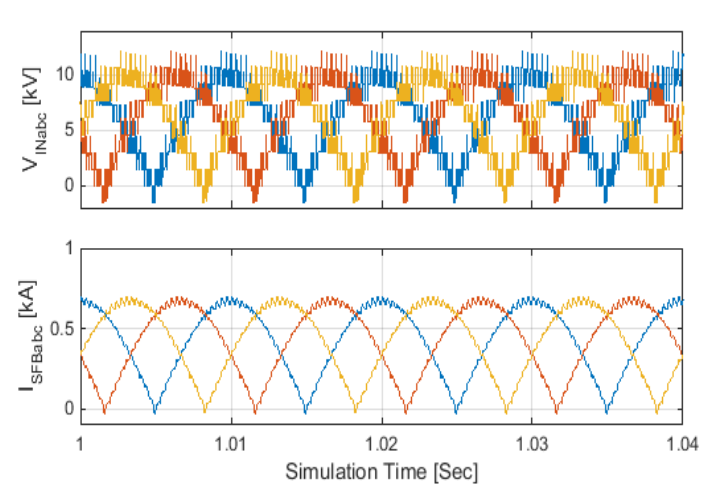

Fig. 13: Voltages and currents at the DC input of the Converter
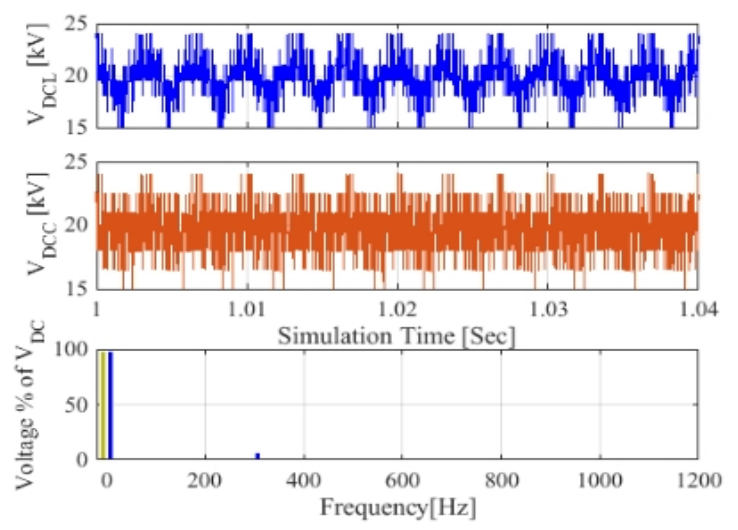

Fig. 15: Filtered and DC side voltages and the FFT of the filtered voltage

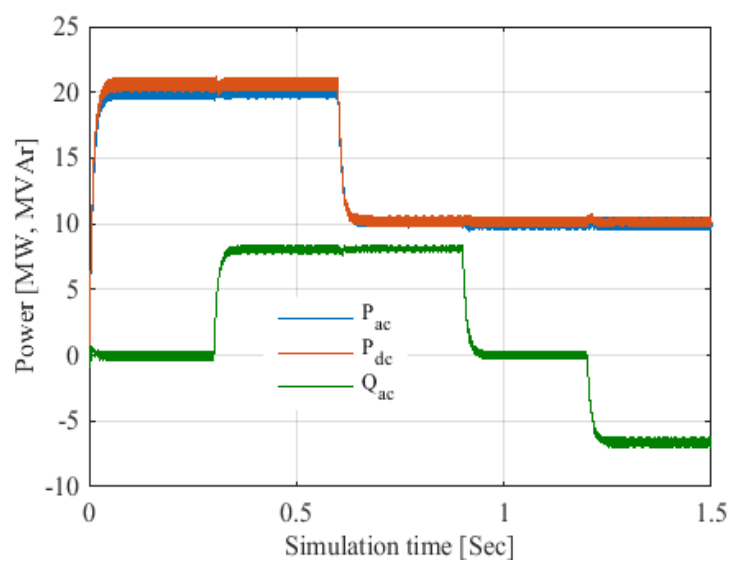

Fig. 17: Power transferred between the ESBC and the DC and AC network submodules in the CL, SFB and T-branch arms respectively. It can be observed that the operation of the ESBC produces high quality current waveforms and can also produce good quality AC voltage waveforms with the appropriate number of submodules. In Fig. 13 the voltage imposed at the DC bus of the H-bridge front-end and the corresponding currents are presented. The voltage waveforms formulated by the various arms of the converter to ensure that the converter synthesises 
the right voltages in Fig. 13 and ripple free DC voltage and current are presented in Fig. 14. In Fig. 15, the voltages across the three CLs and that across the DC bus after the T-branch the action are presented. The $\mathrm{T}$-branch removes all the expected voltage harmonics from the DC bus voltage. Also, local capacitor voltages on the submodules in the arms of the various arms are presented in Fig. 16 during the power transients shown in Fig. 17. It is shown that the inter-arm energy management and the intra-arm controllers developed do support the operation of the converter and formulate high quality voltage and current waveforms at the $\mathrm{AC}$ and $\mathrm{DC}$ sides guarantee energy control.

\section{CONCLUSION}

In this paper, an enhanced H-bridge front-end modular multilevel converter has been presented which has the advantage of using significantly fewer submodules and therefore less energy storage than other topologies with similar performances. The proposed converter uses an optimum number of half bridge and full bridge submodules to achieve active and reactive power control and DC voltage active filtering. The operating principle of the converter, the voltage waves shaping and internal energy management principle have been discussed. Results from a scaled down medium voltage demonstrator have been presented to validate the concept of converter operation and the capabilities of the converter.

\section{REFERENCES}

[1] R. Feldman, M. Tomasini, E. Amankwah, J. C. Clare, P. W. Wheeler, D. R. Trainer, et al., "A Hybrid Modular Multilevel Voltage Source Converter for HVDC Power Transmission," Industry Applications, IEEE Transactions on, vol. 49, pp. 15771588, 2013.

[2] S. Allebrod, R. Hamerski, and R. Marquardt, "New transformerless, scalable Modular Multilevel Converters for HVDC-transmission," in Power Electronics Specialists Conference, 2008. PESC 2008. IEEE, 2008, pp. 174-179.

[3] M. M. C. Merlin, T. C. Green, P. D. Mitcheson, D. R. Trainer, R. Critchley, W. Crookes, et al., "The Alternate Arm Converter: A New Hybrid Multilevel Converter With DC-Fault Blocking Capability," Power Delivery, IEEE Transactions on, vol. 29, pp. 310-317, 2014.

[4] G. P. Adam, Finney, S. J., Williams, B. W.,Trainer, D. R.,Oates, C. D. M.,Critchley, D. R., "Network fault tolerant voltage-sourceconverters for high-voltage applications," in $A C$ and $D C$ Power Transmission, 2010. ACDC. 9th IET International Conference on, 2010, pp. 1-5.

[5] G. Bopparaju, "VSC based FACTS and HVDC: ABB's experience," in Sustainable Energy and Intelligent Systems (SEISCON 2011), International Conference on, 2011, pp. I-2-I-2.

[6] L. B. Feng Wang, Tuan Le, "An Overview of VSC-HVDC : State of - art and potential applications in Electric Power Systems," Cigre, 2011.

[7] J D Ainsworth, M Davies, P J Fitz, K E Owen, and D. R. Trainer, "A static var compensator (STATCOM) based on single phase chain circuit convertors," IEE Proceedings, Generation, Transmission and Distribution, vol. 145, July 1998.

[8] E. Amankwah, A. Costabeber, A. Watson, D. Trainer, O. Jasim, J. Chivite - Zabalza, et al., "The Series Bridge Converter (SBC): A
Hybrid Modular Multilevel Converter for HVDC Applications," presented at the EPE 2013, Karlsrue, 2016.

[9] O. F. Jasim and D. R. Trainer, "Voltage Source Converter," EP2858231A1, 2015. 